\title{
Méthodes de topologie qualitative: applications à l'étude des cheminées d'équilibre
}

\author{
Qualitative topology methods: \\ their applications to surge tank design
}

PAR L. SIDERIADÈS,

MAITRE DE CONFÉRENGES A LA FAGULTÉ DES SGrences DE MARSEILLE

\begin{abstract}
On étudie le problème des cheminées d'équilibre dans le cas du nontage hydraulique clássique comportant d'amont en aval un réservoir dont le niveau est considéré comme fixe, une galerie d'amenée débouchant dans la cheminée d'équilibre a la base de laquelle se situe la conduite forcée reliée aux turbines. Il s'agit essentiellement d'un problème non linéaire de vibrations, en ce qui concerne la masse d'ean de la cheminée, et on recherche la section critique de la cheminée. séparant-les oscillations stables des oscillations instables, sachant que cette section est celle de Thoma dans le cas linéaire. Les méthodes de l'analyse topologique qualitative permettent d'obtenir une information assez poussée du systeme hydraulique dans son fonctionnentent en tant qu'oscillaleur non linéaire, pouvant revêtir un aspect varié par le jeu des asservissements. On étudie successivement les oscillations libres, l'asservissement classique pour lequel la section de Thoma apparât comme section minimale d'un ensemble, et enfin un asservissement nouveau où, par rapport au précédent, il est tenu compte de la perte de charge dize a la conduite forcée. Les résultats theoriques de ce dernier cas se révelent intéressants (possibilité de fonctionnement stable avec une cheminée de section pratiquement nulle), et montrent une analogie possible avec les systèmes électroniques.
\end{abstract}

\begin{abstract}
The case of a conventional hydraulic layout for a surge tank system, is considered, with, from apstream to downstream, a reservoir with an assumed constant level, a supply tunnel discharging into the surge tank, and a penstock leading from the bottom of the surge tant to the turbines. Where the mass of water in the surge tank is concerned, the problem is essentially one of non-linear vibrations, involving the determinalion of the critical surge tank cross-section at which separation occurs between stable and unstable oscillations, it being Jnown that this cross-section is the same as that Thoma gives for the linear case. By applying the qualitative topological analysis methods described, it is possible to obtain fairly comprehensive information on the behaviour of the hydraulic system as a non linear oscillalor, the pattern of which may vary depending on feedback effects, The following are considered: 1) free oscillations, 2) the classical form of feedback, for which Thoma's cross-section represents the minimum cross-section for the arrangement, and 3) a new form of feedback in which, contrary to the conventional one, penstock losses are allowed for. The theorelical results obtained for the latter are interesting, as they point to the possibility of stable operation wilh a surge tank having an infinitely small cros-section, as well as showing a possible analogy with electronic systems.
\end{abstract}

\section{INTRODUCTION}

Le problème de la stabilité des chambres d'équilibre s'est posé pour la première fois en 1904, peu après la construction de l'usine d'Heimbach (groupe des installations de la Ruhr) qui comportait, d'amont en aval, suivant le schéma simplifié de la figure 1, un lac artificiel, un ouvrage de prise d'eau, une galerie d'amenée débouchant dans une cheminée d'équilibre d'où partaient deux conduites forcées alimentant six turbines Francis qui, sous une chute de $70 \mathrm{~m}$, développaient $1500 \mathrm{ch}$ chacune.
Lorscue le niveau du lac réservoir était bas à la fin de l'été, le niveau de l'eau dans la cheminée d'équilibre oscillait lentement, faisant varier la pression aux machines de 90 à $110 \mathrm{~m}$ et, pour maintenir constante la puissance fournie par la centrale, les régulateurs ouvraient et fermaient la turbine suivant le niveau variable dans la cheminée.

En 1910, le professeur Thoma a traité le problème d'une manière qui est considérée aujour- 


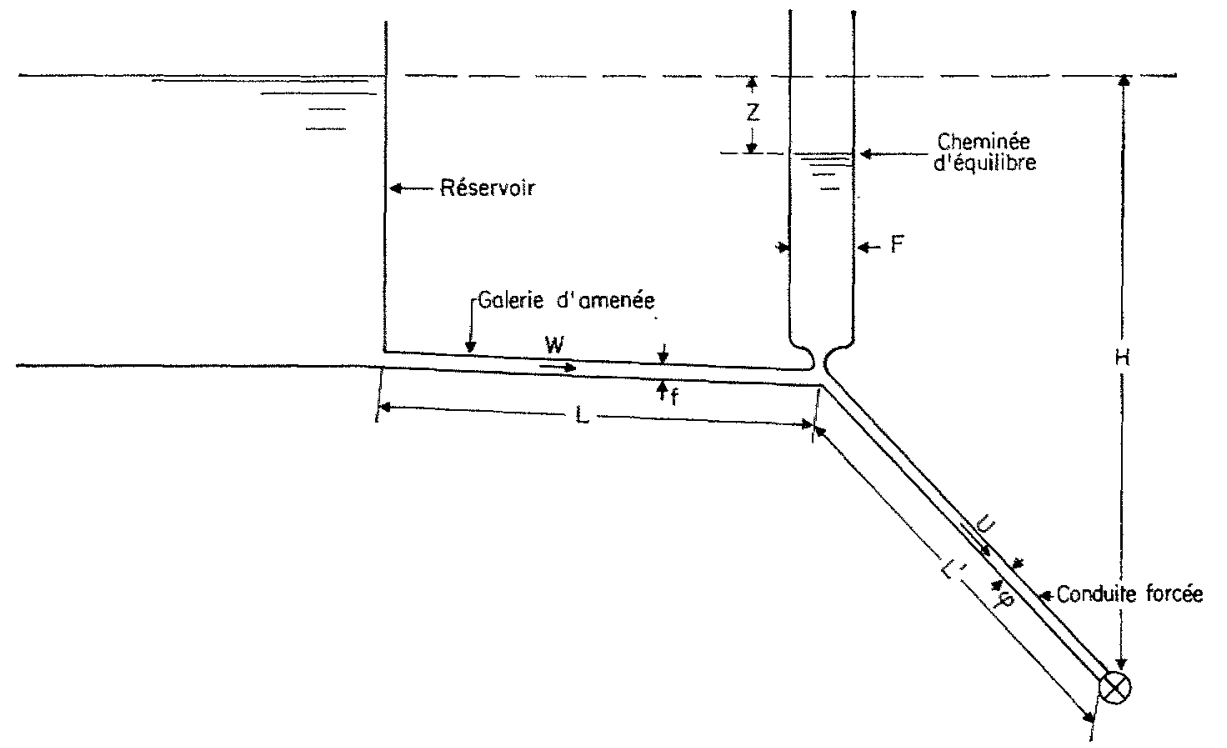

Fig. 1

Schéma simplifié de l'installation hydraulique. d'hui comme classique $[1]$. Et depuis, de nombreux ingénieurs et savants éminents se sont intéressés à ce problème capital pour les installations hydro-électriques, et de très nombreux articles et ouvrages ont été écrits à ce sujet.

Tout récemment, en décembre 1959, au cours d'une réunion de l'American Society of Mechanical Engineers, à New-Jersey, le docteur C. Jaeger a mis l'accent sur la nécessité de définir, pour toute section adoptée pour une cheminée d'équilibre, un facteur de stabilité [2]. Car, depuis l'étude de base de Thoma, qui a le premier déterminé théoriquement une section critique séparant les oscillations stables des oscillations instables, un grand nombre d'études théoriques ef de vérifications expérimentales lant au laboratoire qu'au chantier, ont montré pertinemment que, suivant les cas, on peut s'écarter franchement dans le sens d'une diminution de la section de Thoma (exemple de Jouques), ou au contraire, être forcé de s'y maintenir et, même de majorer cette section (exemple de Cordéac).

Le problème est loin d'être simple, et il apparait que les lois définissant les pertes de charge ainsi que les divers asservissements utilisés jouent un rôle primordial, comme on pourra le constater dans ce qui suit. Mais nous pensons que pour bien comprendre le problème des cheminées d'équilibre, utilisées dans la pratique avec asservissements, il est nécessaire, comme l'avait déjà préconisé $M$. Ailleret en 1957, à la suite d'une communication de MM. Chevalier et Hug [3], d'étudier une centrale juste à la limite de l'instabilité, ou même mieux, en régime libre, indépendamment de tout asservissement. C'est un problème que les méthodes de l'analyse topologique qualitative permettent de résoudre, et l'information apportée par cette première étude permet d'éclairer la difficulté due à la non-linéarité lorsque interviennent ensuite les asservissements.

\section{I. - OSCILLATIONS LIBRES}

La première nécessité est de préciser les pertes de charge qui interviennent pour les masses d'eau couplées entre elles par le noud hydraulique raccordant la galerie d'amence, la cheminée d'équilibre et la conduite forcée. Ces pertes de charge sont quadratiques, et en supposant qu'en régime variable (cela est une approximation) les relations qui les définissent sont les mêmes qu’en régime permanent, on trouve pour la masse d'eau de la galerie une perte quadratique $\mathrm{PW}^{2}$ telle que :

$$
2 g \mathrm{P}=(1+\zeta)+\left(\frac{f^{2}}{\varphi^{2}}-1\right) \cos ^{2} \omega
$$

et pour la masse d'eau de la conduite forcée une perte quadratique $\lambda \mathrm{Q}^{2}$ telle que :

$$
2 g f^{2} \lambda=\zeta^{+}+\left(\frac{f^{2}}{\varphi^{2}}-1\right) \sin ^{2} \varphi
$$


avec :

$\zeta$ : Perte de charge dans la galerie d'amenée;

$\xi$ : Perte de charge dans la conduite forcée et $\xi^{+}=\frac{f^{2}}{\varphi^{2}} \xi$;

(1) : Paramètre de couplage;

$f, \varphi$ : Sections de la galerie d'amenée et de la conduite forcée.

Les équations régissant le système hydraulique sont déterminées

- par l'équation de continuité :

$$
f \mathrm{~W}=\mathrm{FV}+\mathrm{Q} \quad \text { avec } \mathrm{V}=d \mathrm{Z} / d t
$$

- par l'application du théorème de l'énergie cinétique aux masses d'eau de la galerie d'amenée et de la conduite forcée :

$$
\left\{\begin{array}{l}
\frac{\mathrm{L}}{g} \frac{d \mathrm{~W}}{d t}+\mathrm{Z}+\mathrm{PW}=0 \\
\frac{\mathrm{L}^{\prime}}{g^{\prime}} \frac{d \mathrm{Q}}{d t}+\lambda \mathrm{Q}^{2}-\mathrm{H}-\mathrm{Z}=0
\end{array}\right.
$$

On trouve ainsi un système dynamique équivalent :

$$
\begin{gathered}
\frac{d x}{\mathrm{X}}=\frac{d y}{\mathrm{Y}}=\frac{d z}{\mathrm{Z}}=d t \\
\left\{\begin{array}{l}
\mathrm{X}=-\frac{g}{\mathrm{~L}}\left(z+\mathrm{P} x^{2}\right) \\
\mathrm{Y}=-g \frac{\stackrel{0}{\mathrm{~L}^{\prime}}\left(\lambda y^{2}-z-\mathrm{H}\right)}{\mathrm{Z}=-\frac{1}{\mathrm{~F}^{\prime}}(y \cdots-f x)}
\end{array}\right.
\end{gathered}
$$

en posant :

$$
x=\mathrm{W}, \quad y=\mathrm{Q}, \quad z=\mathrm{Z} .
$$

Les résultats de l'étude topologique montrent qu'il existe un seul point singulier dans le domaine correspondant au fonctionnement physique qu'on peut définir par $Q>0$. Ce point est un nœud-foyer stable (fig. 2). La proprićté

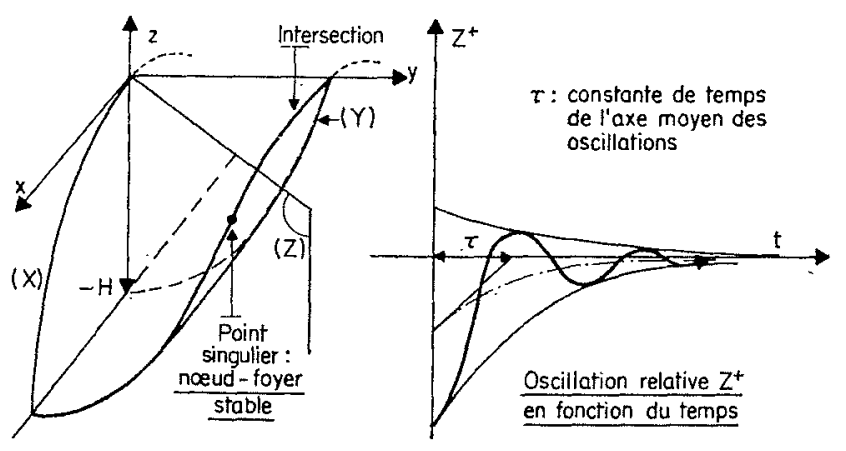

FIG. 2 fondamentale - d'ailleurs physiquement évidente - est que le système est toujours stable; les oscillations sont toujours amorties. On peut faire une étude complète dans le cas très général où :

et :

$$
(\mathrm{P} / \mathrm{L})<<\left(\% f \lambda / \mathrm{L}^{\prime}\right)
$$

$$
(f / L)<<\left(\varphi / L^{\prime}\right)
$$

et montrer que la loi des oscillations est sensiblement de la forme :

$$
Z=A e^{-\beta t} \cos (\omega t+\varphi)+B e^{-\gamma t}
$$

L'axe moyen des oscillations est une courbe exponentielle : c'est une solution réelle qu'on peut toujours vérifier. Il faut cependant remarquer que dans la pratique, la constante de temps de cet axe moyen est très faible. De sorte qu'on peut ramener avec une bonne approximation le problème tridimensionnel à un problème seulement bidimensionnel. Les résultats généraux de cette étude plane, que nous résumons ici, sont les suivants :

-- La période est évidemment supérieure à la période propre $2 \pi(\mathrm{LF} / g f)^{1 / 2}$;

- Il existe un ensemble de valeurs de $F$ pour lesquelles le système stable donne lieu à des oscillations; en dehor's de cette zone, le régime est apériodique. Intuitivement, on peut imaginer qu'un tel régime apériodique existe pour des sections de cheminées très faibles (la cheminée est alors un tube de Pitot), et pour des sections très grandes (la cheminée est alors un grand réservoir). Il est curieux de constater que les deux sections limitant cet ensemble pour lequel les oscillations existent, sont géométriquement inverses dans une inversion dont la puissance est déterminée par une valeur voisine (tantôt supérieure, tantôt inférieure) de la section de Thoma.

-- En régime permanent, la loi de l'écoulement est : $\lambda Q^{2}=\mathbf{H}+Z$. Il est intéressant de remarquer qu'au coefficient $\lambda$ près, cette relation est celle de Torricelli, et que le coefficient $\lambda$ s'exprime simplement en fonction de la section vannée que peut renfermer la conduite forcée. On a en effet :

$$
\lambda=\lambda_{0}+\frac{1-a^{2}}{2 g a^{2} \omega^{2}}
$$

$\varphi$ : Section de la conduite forcée;

a० : Section vannée;

$\lambda_{0}$ : Coefficient de perte de charge pour la conduite forcée seule.

Ce résultat est très utile pour l'application à certains types d'asservissements. 


\section{II. - ASSERVISSEMENT $\mathrm{Q}(\mathrm{H}+\mathrm{Z})=\mathrm{C}^{\text {te }}$}

Le système hydraulique que nous venons d'ctudier présente l'avantage de donner des oscillations qui sont toujours stables, quelle que soit la section de la cheminée d'équilibre. Mais si on examine l'énergie ainsi recueillie en agissant sur une turbine, cette énergie est fluctuante. Pour la stabiliser, on a imaginé un asservissement tel que le produit de la hauteur d'eau totale dans la cheminée par le débit soit constant; ainsi on fixe une énergie voisine (supérieure) de l'énergie potentielle du système. Et théoriquement on peut passer sous silence les pertes de charge dues à la conduite forcée. L'équation différentielle due à la masse d'eau de la conduite forcée est ainsi remplacée par une simple relation, celle dc l'asservissement; et le problème est plan. Mais il est ici essentiellement non linéaire. Et seules les méthodes topologiques permettent de le traiter complètement.

Thoma a montré le premier qu'il existe une section critique de la cheminée séparant les oscillations stables des oscillations instables. Mais l'expérience montre que cette section esi valable lorsque :

- la puissance demandée (valeur de la constante de la relation d'asservissement) est faible;

- la hauteur de chute tolale $\mathrm{H}$ est très grande devant la perte de charge traduite en hauteur d'eau $\mathrm{PW}^{2}$ :

$$
\mathrm{H}>>\mathrm{PW}^{2} \text {. }
$$

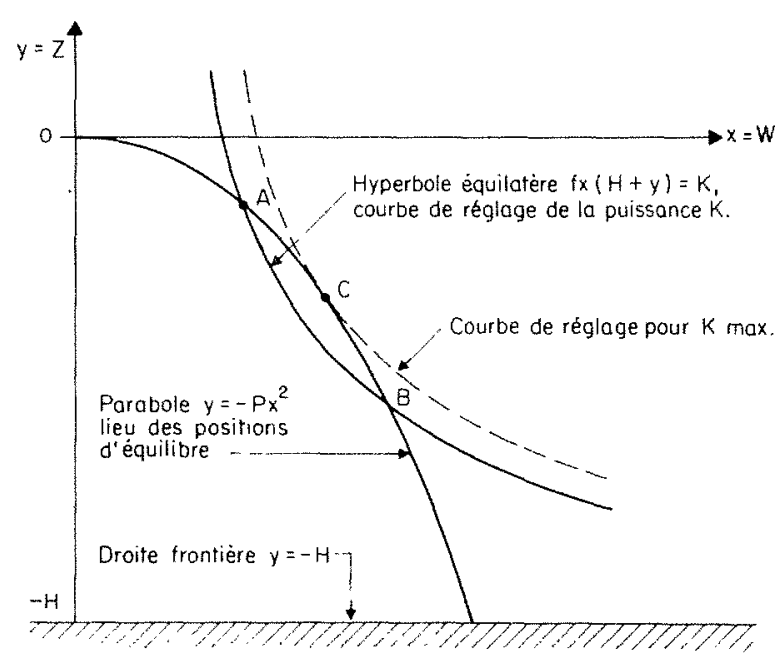

Fig. 3

Disposition graphique des courbes $\mathrm{X}, \mathrm{X}, \mathrm{T}$.
Quand la puissance devient grande, ou que la hautcur de chute devient faible, alors, l'expérience montre que, pour ce type d'asservissement exclusivement, la section critique est toujours supérieure, et parfois notablement, à la section de Thoma.

Nous allons montrer que les résultats du problème non linéaire sont forcément dispersés et que pour ce type d'asservissement, la section de Thoma est une section minimale d'un ensemble.

Les équations sont les suivantes :

$$
\left\{\begin{array}{l}
f \mathrm{~W}=\mathrm{FV}+\mathrm{Q} \\
\frac{\mathrm{L}}{g} \frac{d \mathrm{~W}}{d t}+\mathrm{Z}+\mathrm{PW}^{2}=0 \\
\mathrm{Q}(\mathrm{H}+\mathrm{Z})=\mathrm{K}
\end{array}\right.
$$

En posant $x==\mathrm{W}$ et $y=Z$, on obtient le système dynamique suivant avec courbe frontière [5]:

$$
\frac{d x}{\mathrm{X}}=\frac{d y}{\mathrm{Y}}=\frac{d t}{\mathrm{~T}}\left\{\begin{array}{l}
\mathrm{X}=-\mathrm{F}(\mathrm{H}+y)\left(y+\mathrm{P} x^{2}\right) \\
\mathrm{Y}=\frac{\mathrm{L}}{g}[f x(\mathrm{H}+y)-\mathrm{K}] \\
\mathrm{T}=\frac{\mathrm{LF}}{g}(\mathrm{H}+y)
\end{array}\right.
$$

La discussion topologique fait apparaittre les résultats ci-après :

--- Le système comporte une courbe frontière $\mathrm{T}=0$ qui est la droite $y=--\mathrm{H}$. Cela n'est pas gênant, car la zone de fonctionnement est précisément définie par $y>-\mathrm{H}$;

- La parabole $y=-\mathbf{P} x^{2}$ est le lieu des positions d'équilibre (si elles existent); sa position ne dépend pas de l'énergie utilisée $\mathrm{K}$;

- La courbe de réglage est l'hyperbole équilatère $f x(\mathrm{H}+y)=\mathrm{K}$ dont les asymptotes sont constituées par la droite frontière et l'axe des $y$;

- Ces deux courbes se coupent en général en deux points dont A (fig. 3) est un foyer et $B$ un col. Suivant que le système est stable 


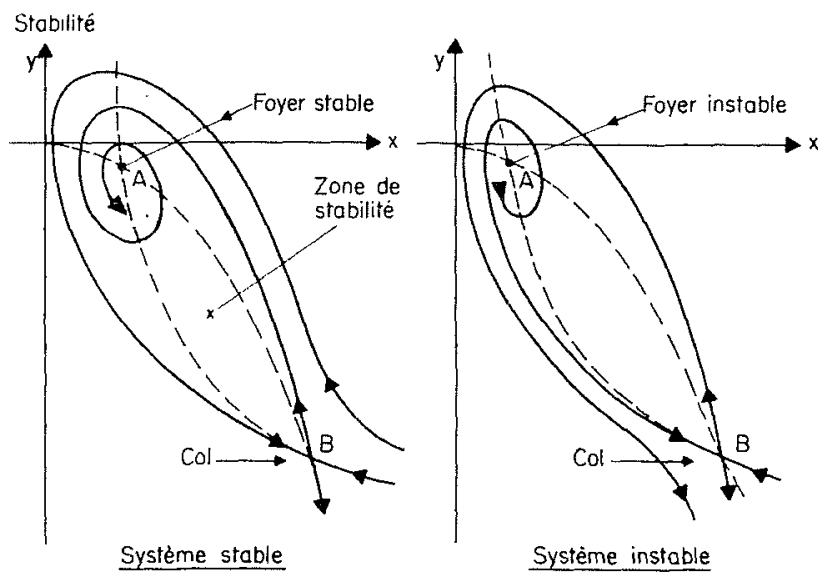

FIG. 4

ou instable, les courbes intégrales présentent la disposition indiquée sur la figure 4 .

Du point de vue de la stabilité, seul le point A peut constituer une position d'équilibre stable si la condition suivante est satisfaite :

$$
\mathrm{F}>\frac{\mathrm{LF}}{2 g \mathrm{P}\left(\mathrm{H}+y_{\mathrm{A}}\right)}
$$

La condition de Thoma correspond à une puissance $\mathrm{K}=0$, donc à $y_{\mathrm{A}}=0$. Lorsque la puissance $K$ croît, le point $A$ tend vers le point $C$ où la parabole et la courbe de réglage correspondant à la puissance maximale sont tangentes. En écrivant que l'équation aux abscisses des points d'intersection a une racine double, on obtient la valeur maximale de $\mathrm{K}$ et la cote minimale du point $\mathrm{A}$ confondu alors avec $\mathrm{C}$.

$$
\left\{\begin{array}{l}
\mathrm{K}_{\mathrm{H}}=0,38 \mathrm{fH}(\mathrm{H} / \mathrm{P})^{1 / 2} \\
\mathrm{Y}_{\mathrm{C}}=-\mathrm{H} / 3
\end{array}\right.
$$

Lorsque A est en C, le système est à la limite de l'instabilité, et la section critique maximale de la cheminée est :

$$
\mathrm{F}_{\mathrm{CM}}=\frac{3 \mathrm{~L} f}{4 g \mathrm{PH}}
$$

soit $3 F_{\mathrm{C}} / 2$ en désignant par $F_{\mathrm{O}}$ la section de Thoma.

De sorte que suivant la valeur de la puissance $K$ comprise entre 0 et $K_{M}$, il existe une section critique de la cheminée comprise entre la section de Thoma et notre section maximale $\mathrm{F}_{\mathrm{CM}}$. C'est là l'un des aspects du problème non linéaire que constituent les cheminées, et dont les caractères essentiels le rattachent au problème plus général des oscillateurs non linéaires. Mais il existe un autre aspect de cette non linéarité dont l'explication est purement topologique.

Le point singulier correspondant des courbes intégrales (dans le plan $\mathrm{W}, \mathrm{Z}$ ) est alors un centre et le cycle limite de la théorie de Poincaré [4] est réduit à ce point. Dans son voisinage, les courbes intégrales sont celles d'un foyer répulsif : le système est donc en fait instable et il est impossible d'observer physiquement les oscillations. Pour observer des oscillations stables d'une certaine amplitude, il est nécessaire de mettre en évidence un cycle limite instable enfermant une zone au centre de laquelle le point singulier devient alors un foyer stable. En d'autres termes, il est nécessaire de majorer la section critique précédemment obtenue. Suivant les travaux de Marris [6] appliquant les méthodes d'approche de Kryloff et Bogoliuboff [7] et d'après des résultats personnels, nous avons adopté un coefficient moyen de majoration égal à 1,15. De sorte que la section critique maximale devient alors égale à 1,72 fois la section de Thoma.

Nous avons appliqué ces résultats à l'exemple (fig. 5) mentionné par Marris [6] concernant les

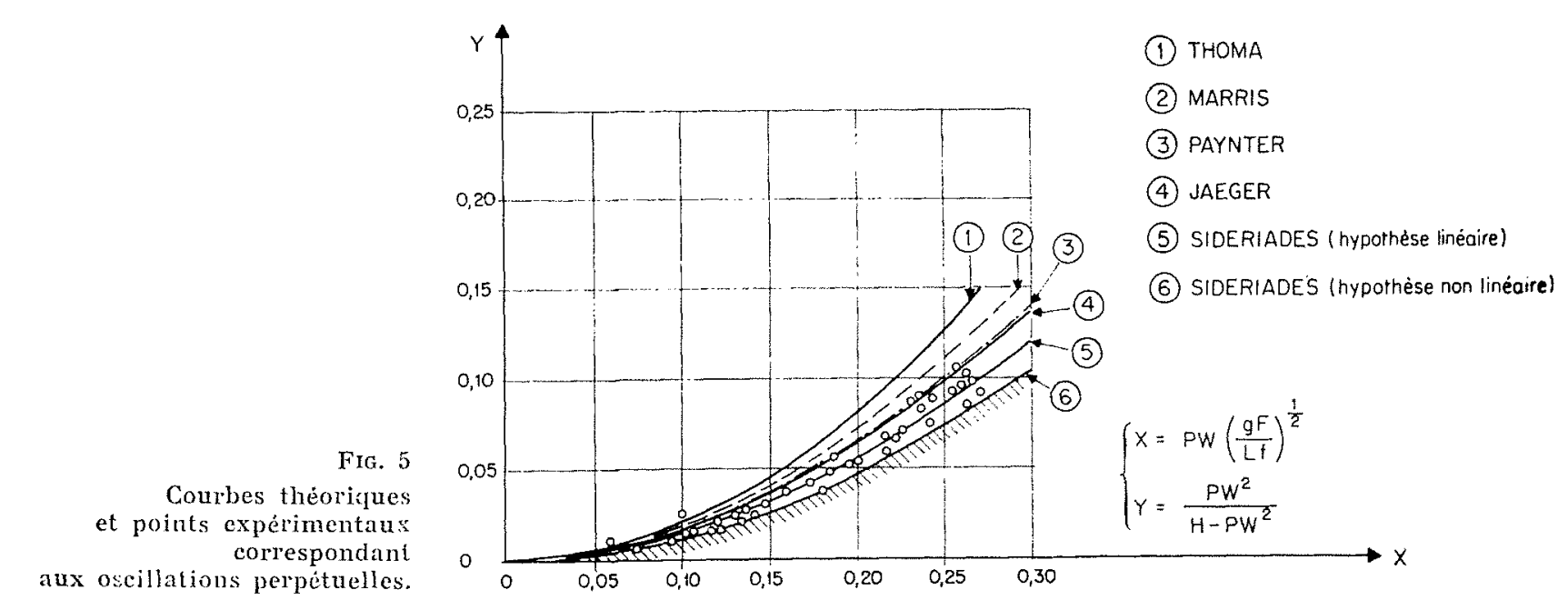


valeurs expérimentales régissant les oscillations perpétuelles dans le plan :

$\mathrm{X}=\mathrm{PW}(g \mathrm{~F} / \mathrm{L} f)^{1 / 2}, \quad \mathrm{Y}=\mathrm{PW}^{2} /\left(\mathrm{H}-\mathrm{PW}^{2}\right)$, et leur comparaison avec les courbes théoriques proposées par Marris, Paynter et Jaeger [8]. Il apparaît nettement que la rectification due à la présence d'un cycle limite n'agit que sur la section de Thoma en tant que section minimale d'un ensemble, donc égale à $\mathrm{F}_{\mathrm{O}}$ : la plupart des points expérimentaux sont situés en dehors des courbes proposées. Si on applique ensuite la rectification non linéaire suivant le double aspect que nous avons envisagé, nous obtenons deux nouvelles courhes sur cette même figure :

- La courbe notée $\mathrm{n}^{\circ} 5$ relative à la section $3 \mathrm{~F}_{\mathrm{C}} / 2$ avec cycle limite ponctuel;
- La courbe notce $n^{\circ} 6$ relative à la section majorée $1,72 \mathrm{~F}_{\mathrm{C}}$ avec cycle limite effectif : tous les points expérimentaux sont situés audessus de cette dernière courbe.

On notera également la validité de la section de Thoma Iorsque la puissance $K$ est faible. Alors, à cause de la courbure de la parabole au voisinage de son sommet (A en 0 ), qui se présente un peu comme une courbe de résonance en électricité, «l'affaiblissement », c'est-à-dire la cote de $y_{\mathrm{A}}$ est à peu près nulle; et tout se passe, du point de vue de la stabilité, comme si le système était linéaire. Mais dès que la cote de $y_{\mathrm{A}}$ n'est plus négligeable, alors la section de Thoma détermine un système instable, comme on peut le voir sur la figure 6 oú on a tracé les courbes intégrales d'un système possédant une

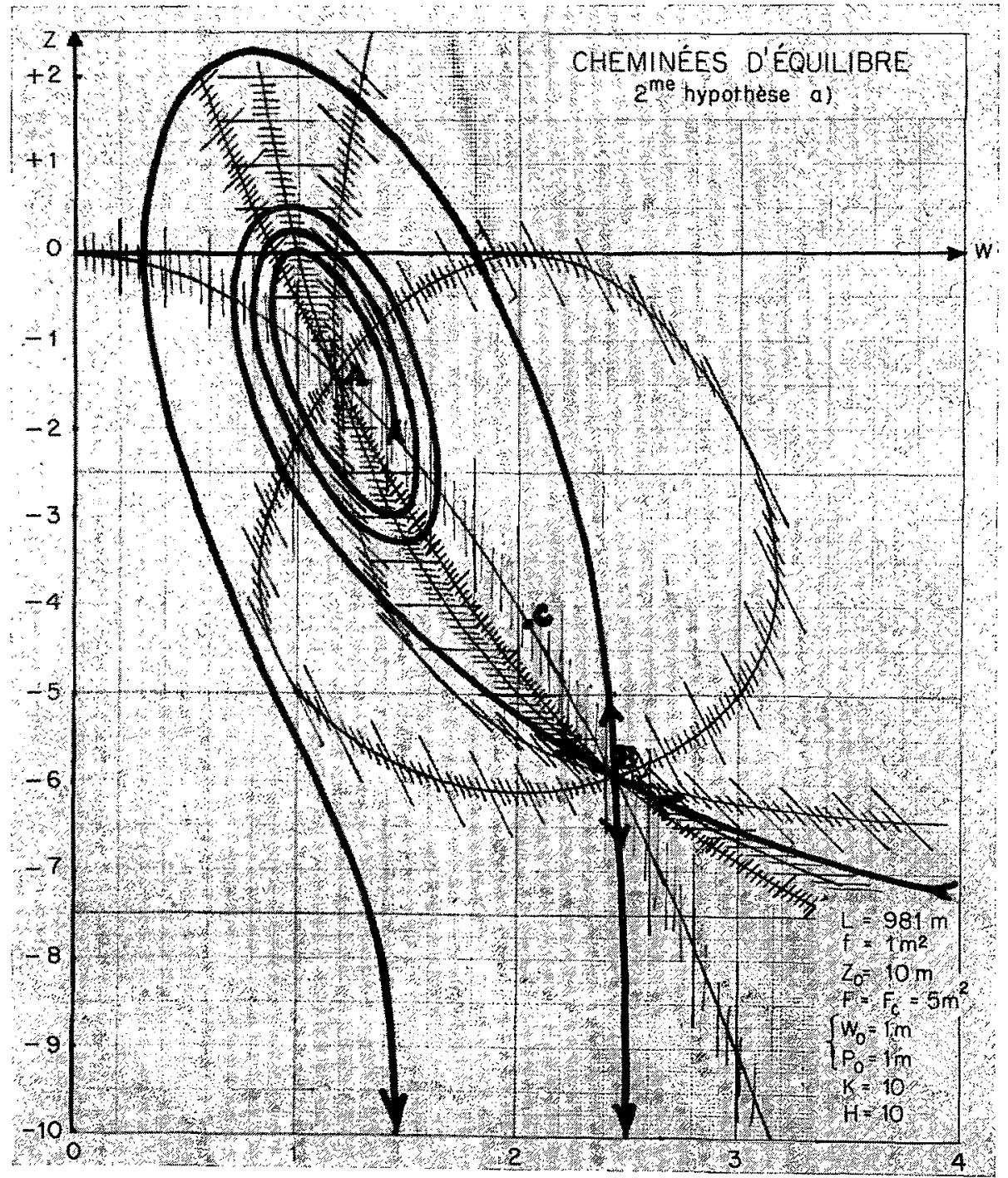

FIG. 6 


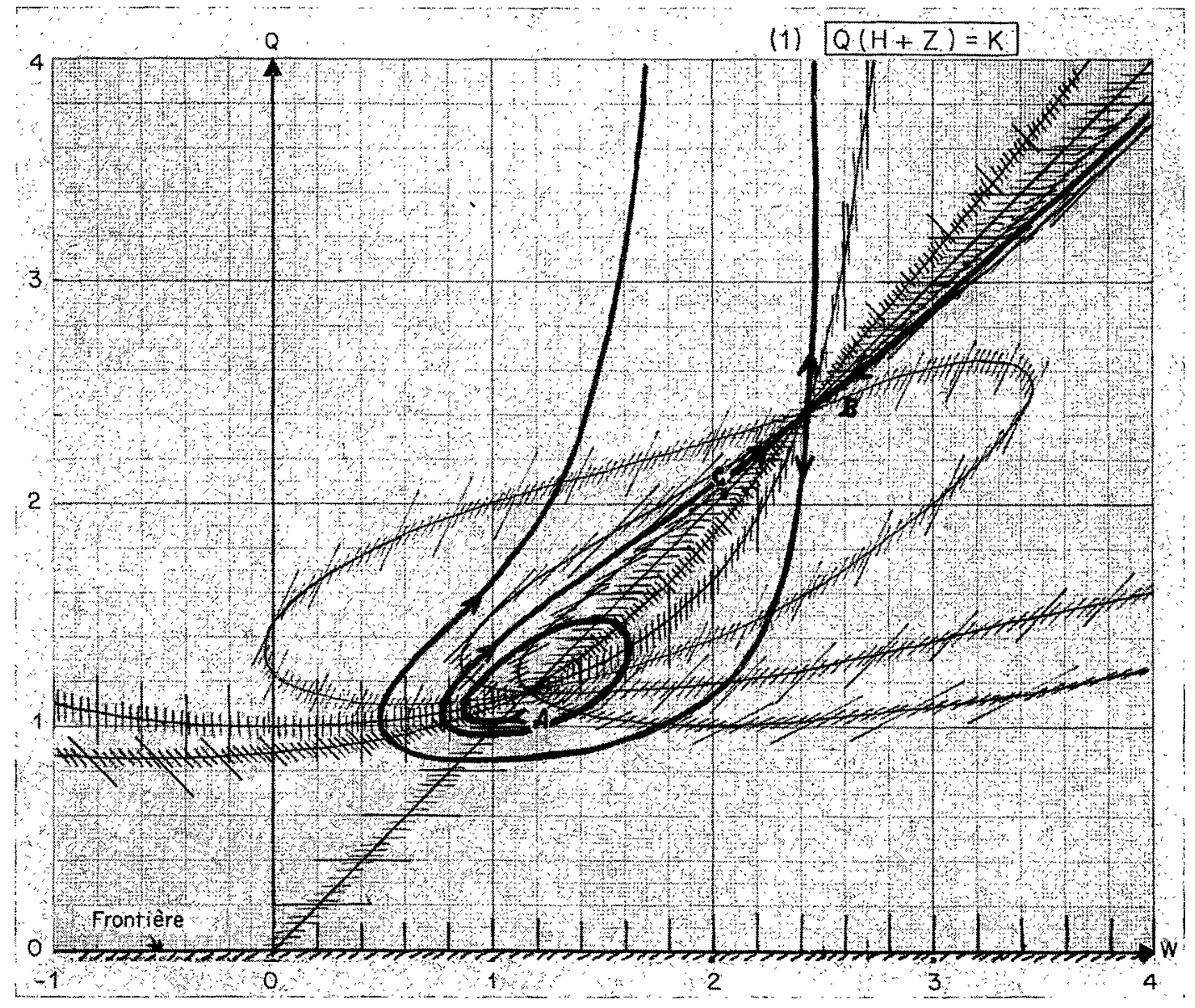

FIG. 7

section de Thoma, mais dont la puissance d'utilisation est loin d'être négligeable dans le plan W, Z. La figure 7 représente les courbes inté- grales du même système dans le plan W, Q. Ce dernier plan sera très utile à considérer pour le type suivant d'asservissement.

\section{III. - ASSERVISSEMENT $\mathrm{Q}\left(\mathrm{H}+\mathrm{Z}-\lambda \mathrm{Q}^{2}\right)=\mathrm{K}$}

L'étude de l'asservissement précédent montre pertinemment que la stabilité est assurée moyennant des sections de cheminées supérieures à la section de Thoma. Or des installations fonctionnent d'une manière stable avec des sections bien inférieures à celle de Thoma; par exemple à Jouques, la section de Thoma serait de $650 \mathrm{~m}^{2}$ et l'installation fonctionne avec trois cheminées en parallèle de $50 \mathrm{~m}^{2}$ chacune, soit un total de $150 \mathrm{~m}^{2}$. Il n'y a aucune contradiction avec les résultats que nous venons de présenter; seul le type d'asservissement envisagé, qui peut être plus ou moins complexe, est différent. Nous allons exposer, dans ce qui suit, les résultats d'étude topologique du système où on asservit à demeurer constant le produit du débit de la conduite forcée (comme dans le cas précédent) par la hauteur d'eau $\left(\mathrm{H}+Z-\lambda \mathrm{Q}^{2}\right), \lambda$ étant un paramètre de perte de charge que nous avons précédemment défini (à la différence du cas précédent, cette hauteur d'eau serait l'indication d'un tube de Pitot placé à l'extrémité inférieure de la conduite forcée).

Les équations sont les suivantes :

$$
\left\{\begin{array}{l}
f \mathrm{~W}=\mathrm{FV}+\mathrm{Q} \\
\frac{\mathrm{L}}{g} \frac{d \mathrm{~W}}{d t}+\mathrm{Z}+\mathrm{PW}^{2}=0 \\
\mathrm{Q}\left(\mathrm{H}+\mathrm{Z}-\lambda \mathrm{Q}^{2}\right)=\mathrm{K}
\end{array}\right.
$$


En posant $x=\mathrm{W}$ et $y=\mathrm{Q}$, on obtient le système dynamique suivant avec courbe frontière :

$$
\frac{d x}{\mathrm{X}}=\frac{d y}{\mathrm{Y}}=\frac{d t}{\mathrm{~T}}
$$

$$
\left\{\begin{array}{l}
\left.\mathrm{X}=-\mathrm{F}(\mathrm{K}-2 \lambda y)^{3}\right)\left(\mathrm{P} y x^{2}+\lambda y^{3}-\mathrm{H} y+\mathrm{K}\right) \\
\mathrm{Y}=-\frac{\mathrm{L}}{g} y^{3}(f x-y) \\
\mathrm{T}=\frac{\mathrm{FL}}{g} y\left(\mathrm{~K}-2 \lambda y^{3}\right)
\end{array}\right.
$$

La discussion topologique fait apparaitre les résultats ci-après :

- Le système est fortement non linéaire et comporte une courbe frontière qui se décompose suivant les deux droites :

$$
y=0 \text { et } y=(K / 2 \lambda)^{1 / 3} \text {; }
$$

-- Il existe deux points singuliers $\mathrm{A}$ et $\mathrm{B}$ (cf. fig. 8), séparés par la droite frontière, qui peuvent donner lieu à des positions d'équilibre stable;

- La stabilité est déterminée par le signe de l'expression suivante :

$$
\left(\mathrm{K}-2 \lambda y^{3}\right)\left(2 \mathrm{PF}\left(\mathrm{K}-2 \lambda y^{3}\right)-\frac{\mathrm{L}}{g} f^{2} x\right)
$$

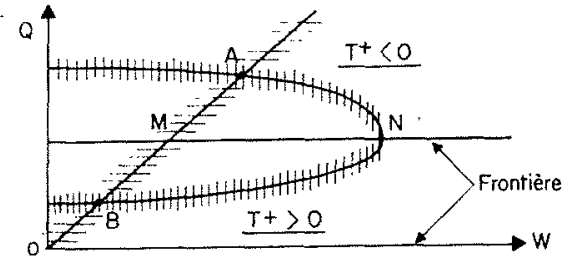

FIG. 8

La discussion dépend des valeurs de $\mathrm{K}$ :

1. Si K est faible (les points $A, M, N$ sont distincts), la condition de stabilité s'écrit :

$$
\mathrm{F}>\frac{\mathrm{L} f^{2} x}{2 g \mathrm{PT}^{+}}
$$

en posant : $\mathrm{T}^{+}=\mathrm{K}-2 \lambda \mathrm{y}^{3}$.

En A cette condition est toujours vérifiée : le système est donc stable quclle que soit la section $F$. Mais la non-linéarité limite cette validité à une zone déterminée du plan $W, Q$ comme on peut le constater sur l'exemple de la figure 9, par un arc de la courbe intégrale aboutissant en $M$ et un segment de la droite frontière passant par $M$.

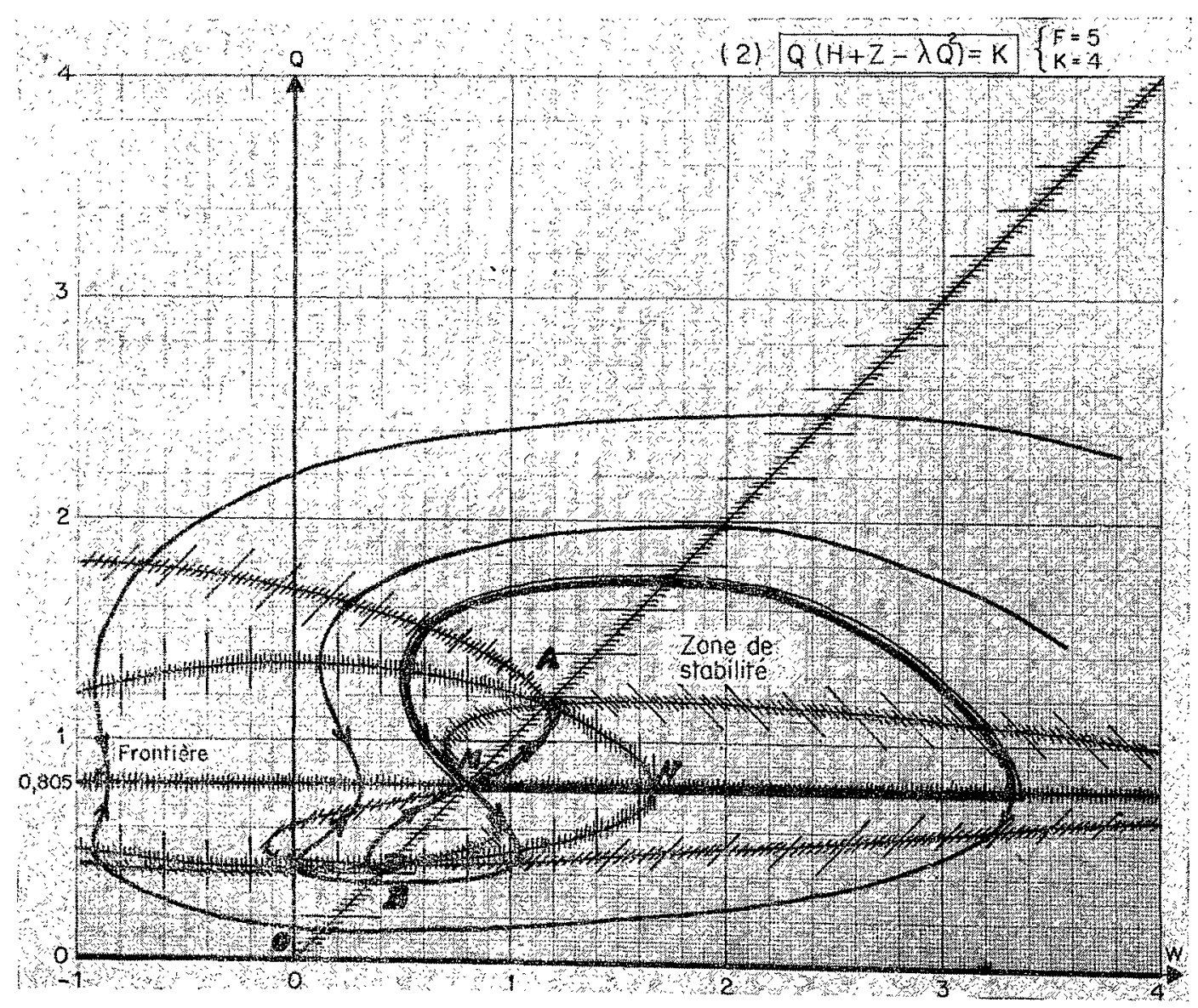

FIG. 9 


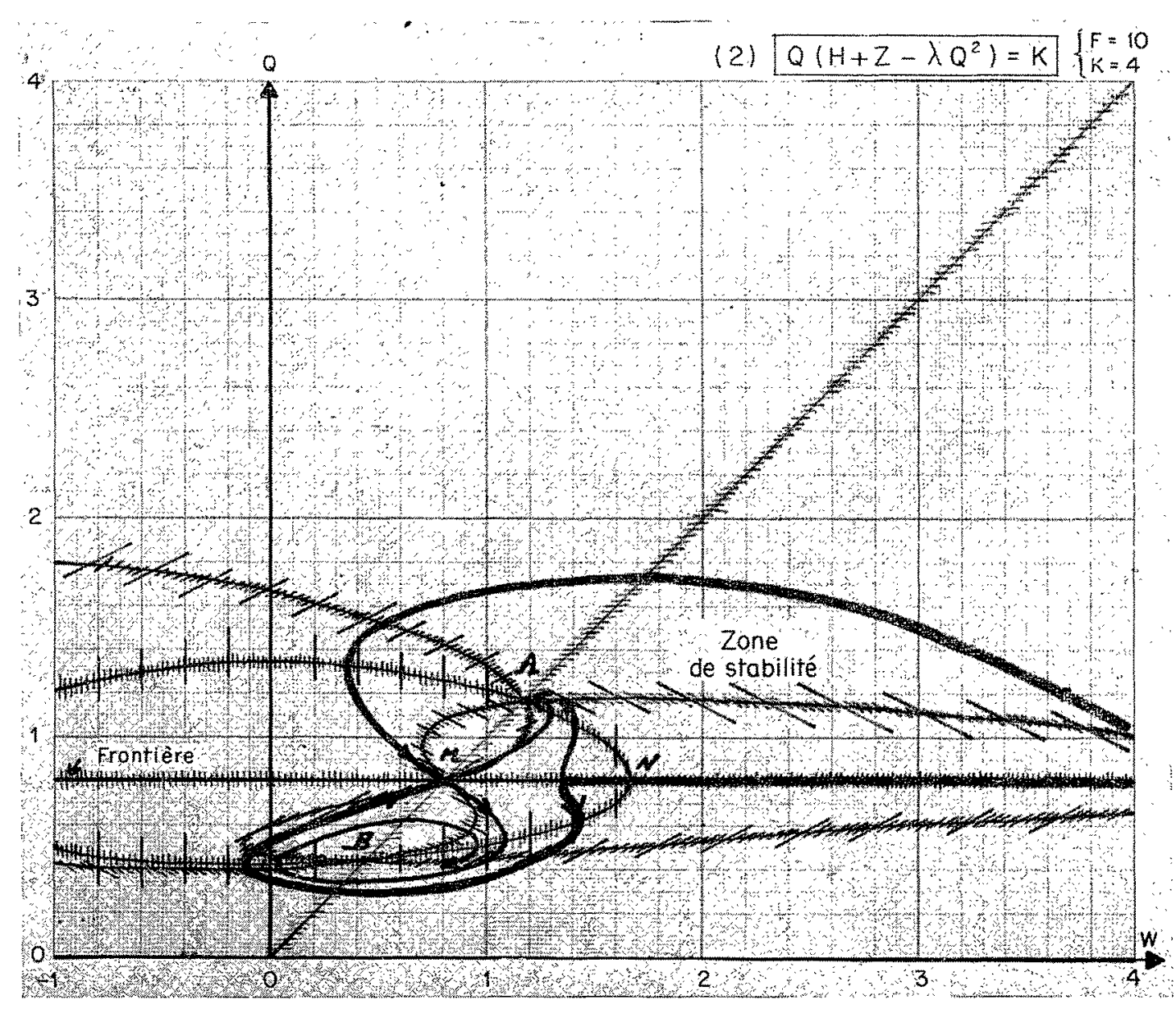

FIG. 10

En B, le système est stable si $F$ est supérieur à une certaine section critique :

$$
\mathrm{F}_{\mathrm{C}}=\mathrm{L} f^{2} x(\mathrm{~B}) / 2 g \mathrm{PT}^{\mathrm{T}}+(\mathrm{B})
$$

et le système est alors bistable comme on peut le constater sur l'exemple de la figure 10 où les deux points $A$ et $B$ sont des foyers attractifs. La non-linéarité limite là encore cette validité à une double zone du plan $W, Q$ (dédoublement de la zone précédente de part et d'autre de la droite frontière).

De sorte que le résultat fondamental est le suivant pour ce premier cas :

Pour $\mathrm{K}$ faible, le système est monostable si $\mathrm{F}$ est inférieur à une certaine valeur critique $F_{C}$, et bistable si $\mathbf{F}$ est supérieur à $\mathbf{F}_{\mathrm{C}}$. II existe une zone du plan W, Q pour laquelle le système est stable quelle que soit la valeur de $F$.

2. $\mathrm{K}_{\mathrm{C} 1}<\mathrm{K}<\mathrm{K}_{\mathrm{C} 2}$ :

En désignant par $K_{C 1}$ la valeur de $K$ pour laquelle les trois points $\mathrm{A}, \mathrm{M}, \mathrm{N}$ sont confondus - et par $K_{C_{2}}$ la valeur de $K$ pour laquelle les deux points $A$ et $B$ sont confondus, on observe dans ce deuxième cas les résultats suivants :

- Le point $A$ est toujours un col, donc instable, -.- Le point $B$ est un foyer stable si $F>F_{C}$.

3. $\mathrm{K}>\mathrm{K}_{\mathrm{C} 2}$ :

Il n'y a plus de point singulier réel et le système est toujours instable quelle que soit la valeur de $\mathbf{F}$.

\section{CONCLUSION}

Les méthodes de l'analyse topologique qualitative se révèlent ainsi comme un instrument mathématique extrêmement intéressant pour l'étude des systèmes oscillants non linéaires, non seulement dans le domaine de l'Hydraulique avec l'exemple des cheminées d'équilibre comme 
on vient de le voir, mais encore dans de nombreux domaines de la Physique, et tout particulièrement en Electronique, en Mécanique et en Acoustique. Le double aspect fondamental de ces méthodes est d'une part dé préciser les singularités du problème physique (ce qui est souvent le plus difficile), et d'autre part d'apporter une information générale sur le fonctionnement non linéaire, en mettant en évidence des aspects insoupçonnés de ce fonctionnement qui permettent de mieux comprendre et d'expliquer la réalité physique souvent complexe.

Mais ces méthodes topologiques sont également un instrument puissant, car elles permettent la synthèse d'un systéme physique inconnu et sa construction effective, réelle, sur la simple prévision de son fonctionnement rendu possible à partir de l'information constituée par le tracé des courbes intégrales. C'est ainsi que nous avons été amené à définir un type d'asservissement qui permettrait de conserver la stabilité du système hydraulique, tout en diminuant fortement la section de la cheminée. La solution qualitative confirme le résultat pressenti, au prix d'une légère perte sur la puissance d'utilisation. De plus ce nouveau type d'asservissement permet de réaliser un oscillateur à deux positions d'équilibre dans certains cas. C'est là un résultat qui était connu avec les oscillateurs électroniques du genre basculeurs. Et il est à prévoir que la porte ainsi ouverte aux analogies ElectroniqueHydraulique dans ce domaine ne manquera pas de susciter dans l'avenir de fructueuses recherches.

\section{ANNEXE}

Etude mathématique des systèmes du premier ordre.

A. Théorite des valeurs propres appliouée AUX POINTS SInguliers. - Soit le système :

$$
\frac{d x}{\mathrm{X}(x, y)}=\frac{d y}{\mathrm{Y}(x, y)}=d \dot{t}
$$

où $\mathrm{X}, \mathrm{Y}$ sont des polynômes en $x, y$, ou plus généralement des fonctions holomorphes de leurs arguments. Considérons la transformation définie par la matrice :

$$
\alpha=\left|\frac{\mathrm{D}(\mathrm{X}, \mathrm{Y})}{\mathrm{D}(x, y)}\right|, \quad \text { soit } a u=\mathrm{S} u,
$$

D désignant le déterminant jacobien, $S$ étant une grandeur scalaire - appliquée au vecteur $u$ en un point singulier (point commun aux deux courbes de base $\mathrm{X}=0$ e! $\mathrm{Y}=0$ ). Les valeurs propres sont les racines de l'équation caractéristique :

$$
\mathrm{S}^{2}+\xi \mathrm{S}+\eta=0
$$

Le plan caractéristique (ou plan des valeurs propres) est commun à tous les systèmes du type (1); la distribution du ou des points singuliers dans ce plan caractérise qualitativement les propriétés du système.

Les directions propres peuvent être obtenues à partir de l'égalité :

(*) On peut remarquer que si l'on écrit l'équation caractéristique sous la forme:

$$
\mathrm{S}^{2}-\left(\mathrm{X}^{\prime}{ }_{x}+\mathrm{Y}^{\prime}{ }_{y}\right) \mathrm{S}+\left(\mathrm{X}_{x}{ }_{x} \mathrm{Y}^{\prime}{ }_{y}-\mathrm{X}^{\prime}{ }_{y} \mathrm{Y}_{z}^{\prime}\right)=0,
$$

l'équation aux directions propres devient :

$$
\mathrm{X}^{\prime}{ }_{y} \lambda^{2}+\left(\mathrm{X}_{\mathbf{x}^{\prime}}-\mathrm{Y}_{\mathrm{y}}^{\prime}\right) \lambda-\mathrm{Y}_{\mathrm{s}}^{\prime}=0 \text {. }
$$

$$
\alpha^{-1} u=|k| u
$$

La matrice a liée à un point singulier fait ainsi apparaître deux couples de grandeurs fondamentales :

a) Les valeurs propres, qui, du point de vue dynamique, jouent le rôle de coefficients de contraction ou de dilatation attachés au point singulier;

b) Les directions propres, qui sont les directions géométriques suivant lesquelles s'exercent ces contractions ou ces dilatations. Etant évidemment dépendantes, elles obéissent à la même condition de réalité, déterminée par le signe de la quantité :

$$
\Phi=\xi^{2}-4 \eta \text {. }
$$

La figure 11 représente cette courbe, qui est une parabole, dans le plan caractéristique.

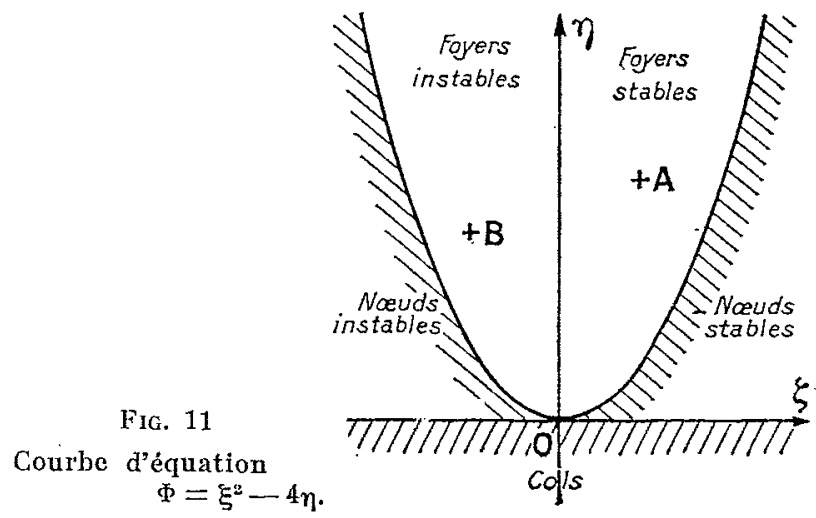




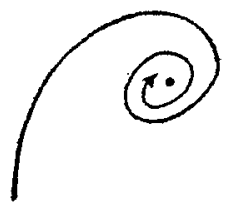

1

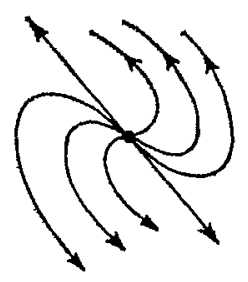

5

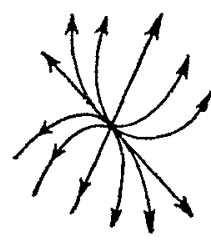

2

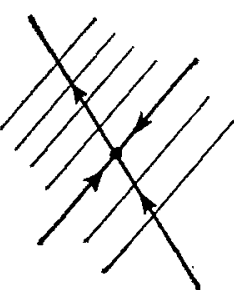

6

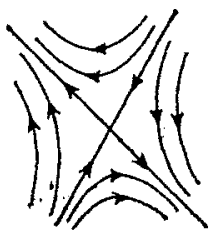

3'

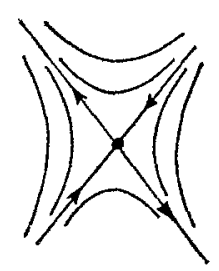

7

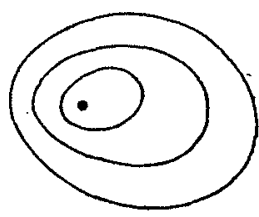

4

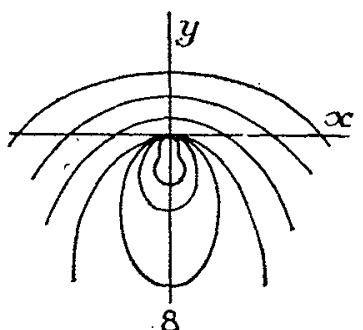

8

Fig. 12

Figuration des divers points singuliers des systèmes non linéaires du premier ordre : 1 , foyer; 2 , nœud; 3 , col; 4 , centre-foyer; 5 , noud-foyer; 6, noud-col; 7, centre-col; 8, nœud-centre-foyer.

Il est ainsi possible de lier à chaque point singulier deux vecteurs $u$ (qui peuvent être imaginaires) définissant un champ propre. Un tel champ détermine complètement la disposition des courbes intégrales dans le voisinage du point singulier.

L'étude de la nature des points singuliers est basée sur celle du signe des racines $\mathrm{S}$ de l'équation (2).

Nous utilisons plus rationnellement le signe des deux quantités $\eta$ et $\Phi$ précédemment définies. On peut ainsi dénombrer huit points singuliers dont la classification s'effectue de la manière suivante :

\section{a) Points à caractère simple :}

$\Phi>0, \eta>0$ : Les racines réelles sont de même signe et déterminent un nœud. Les courbes intégrales sont tangentes à deux directions propres distinctes (analogie parabolique, fig. 12-2).

$\Phi>0, \eta<0$ : Les racines réelles sont de signes contraires et déterminent un col. Les directions propres sont celles de deux intégrales singulières qui seules passent par le point (analogie hyperbolique, fig. 12-3).

$\Phi\langle 0, \eta\rangle 0$ : Les racines sont imaginaires conjuguées et déterminent un foyer. Les courbes intégrales s'enroulent ou se déroulent autour du point à la manière de spirales (analogie elliptique, fig. 12-1).

b) Points à caractère double : L'une des quantités $\xi, \eta$ ou $\Phi$ est nulle.

$\eta=0:$ on a un noud-col (fig. 12-6).

$\Phi=0:$ on a un noud-foyer (fig. 12-5). $\xi=0:$ on a un centre (deux types). Le centre du type elliptique donne lieu à des courbes fermées (fig. 12-4 et 7).

c) Points à caractère multiple : La constitution d'un tel point est la confusion plus ou moins complexe des caractères précédents. On trouve dans cette catégorie les points singuliers essentiels. La figure 12 illustre ce qui précède (fig. 12-8).

B. Comportement des points singuliers aU COURS DE TRANSFORMations. - Considérons un système plus général que (1) et de la forme :

$$
\frac{d x}{\mathrm{X}(x, y)}=\frac{d y}{\mathrm{Y}(x, y)}=\frac{d t}{\mathrm{~T}(x, y)}
$$

Ce système est équivalent à celui des deux équations :

$$
\begin{array}{r}
-\mathrm{D} \dot{x}+\mathrm{B} \dot{y}+\overline{\mathrm{X}}=0 \\
\mathrm{C} \dot{x}-\mathrm{A} \dot{y}+\overline{\mathrm{Y}}=0
\end{array}
$$

dans lesquelles $\dot{x}=\frac{d x}{d t}, \dot{y}=\frac{d y}{d t}$

selon la notation de Newton, avec :

$$
\mathrm{X}=\overline{\mathrm{AX}}+\overline{\mathrm{BY}}, \mathrm{Y}=\overline{\mathrm{CX}}+\overline{\mathrm{DY}}, \mathrm{T}=\mathrm{AD}-\mathrm{BC},
$$

soit encore sous forme matricielle :

$$
[u]=[\mathrm{T}][\tilde{u}],[u]=\left|\begin{array}{l}
\mathrm{X} \\
\mathrm{Y}
\end{array}\right| \text {, et }[\vec{u}]=\left|\begin{array}{l}
\overline{\mathrm{X}} \\
\overrightarrow{\mathrm{Y}}
\end{array}\right|,[\mathrm{T}]=\left|\begin{array}{l}
\mathrm{AB} \\
\mathrm{CD}
\end{array}\right|
$$

On appelle système statique le système :

$$
[\bar{u}]=0
$$


et système dynamique le système $[\boldsymbol{u}]=0$. On peut alors énoncer quelques théorèmes régissant le comportement des points singuliers au cours de transformations.

Théorème 1. - Les points singuliers des courbes intégrales se divisent en deux catégories :

a) Les points d'absorption : ce sont les points communs aux courbes de base. Ils correspondent à un schéma purement * résistif * (indépendant du temps) du système physique. Ils peuvent constituer des positions d'équilibre; l'énergie fournie par les sources d'alimentation s'écoule alors par ces points;

b) Les points transformés : ils résultent de la transformation dut système statique en système dynamique par les éléments \& purement réactifs $\gg$ (dépendants du temps). Is ne peuvent jamais constituer des positions d'équilibre.

Théorème 11 . - Un point d'absorption peut donner lieu, après transformation, à un noud, à un foyer, ou à un col.

Cette propriété résulte de l'égalité :

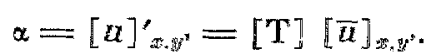

Théoréme III. - Toute position d'équilibre stable satisfait aux conditions simultanées :

$$
\left.\begin{array}{l}
\mathrm{X}=\mathrm{Y}=0, \\
(\mathrm{AD}-\mathrm{BC})\left(\mathrm{X}_{x}^{\prime}+\mathrm{Y}_{y^{\prime}}^{\prime}\right) \\
\quad \leqslant 0 \leqslant(\mathrm{AD}-\mathrm{BC})\left(\overline{\mathrm{X}}_{x}^{\prime} \overline{\mathrm{Y}}_{y^{\prime}}^{\prime}-\overline{\mathrm{X}}_{y^{\prime}}^{\prime} \overline{\mathrm{Y}}_{x}^{\prime}\right)
\end{array}\right\}
$$

Théoreme $1 V$. - Toute transformation orthogonale modifie la nature des nouds et des foyers et conserve les cols.

A partir de l'équation différentielle:

$$
\mathrm{Y}-y^{\prime} \mathrm{X}=0
$$

un système de trajectoires orthogonales est donné par l'équation $\mathrm{X}+y^{\prime} \mathrm{Y}=0$.

Une telle transformation n'altère pas le produit des valeurs propres.
Théorème $V$. - La condition nécessaire et suffisante pour qu'un point d'absorption donne lieu à deux points pseudo-orthogonaux dans deux transformations $[\mathrm{T}]$ et $[\mathrm{T}+]$ est que les déterminants de $[\mathrm{T}]$ et $[\mathrm{T}+]$ soient opposés.

D'après le précédent théorème (4), la transformation orthogonale n'altère pas le caractère essentiellement instable, du point de vue de l'équilibre, qui caractérise les cols. Il est donc intéressant de définir une autre transformation permettant d'obtenir une position stable à partir d'une position instable, c'est-à-dire permettant le passage d'un col à un noud ou à un foyer, et réciproquement. Une telle transformation, modifiant la nature des nouds, des foyers et des cols, est d'un caractère plus général que la transformation orthogonale : on lui a donné le nom de transformation pseudo-orthogonale.

Le, théorème énoncé est basé sur l'égalité :

$$
\alpha=[\mathrm{T}] \bar{\alpha} .
$$

\section{BIBLIOGRAPHIE}

[1] D. Thoma. - Zur Theorie des Wasserschlosses bei selbstätig geregelten Turbinen, Oldenburg, München, 1910.

[2] C. JAEGER. - «A review of surge-tank stability criteria », Trans. A.S.M.E., Series D, Journal of Basic Engineering, Dec. 1960.

[3] J. Chevalier et M. Hug. - La Houille Blanche, $\mathrm{n}^{\circ}$ 6, déc. 1957.

[4] H. Polncaré. - « Sur les courbes définies par une équation différentielle $\gg$. Journal de Mathématiques Pures et Appliquées (1881-1886) et «Méthodes nouvelles de la Mécanique céleste », Gauthier-Villars, Paris, t. I.

[5] L. Sideriades. - « Méthodes topologiques appliquées à l'Electronique $»$. Publications Scientifiques et Techniques da Ministère de l'Air, $\mathrm{n}^{\circ}$ N.T.84, 1959.

[6] A.W. Marris. - «Large water-level displacements in simple surge-tanks», Trans. A.S.M.E., Series D, Journal of Basic Engineering, Dec. 1959.

[7] N. Misonsky. - "Introduction to non linear Mechanies », J. W. Edwards, Ann. Arbor, Michigan (1947).

[8] H. M. PAynter. - «Surge and water hammer problems », Trans. A.S.C.E., Electrical Analogies and Electronics Computers Symposium, vol. 118, 1953, et discussion de H.M. Payner aux deux articles précédents de C. JaEger et A. W. Marris. 